\title{
INFLATIONARY UNIVERSE MODELS AND CLUSTERING OF QUASAR RED SHIFTS
}

\author{
C.Sivaram \\ Indian Institute of Astrophysics \\ Bangalore 560034 \\ India
}

ABSTRACT. Recently it has been shown that many of the puzzling features of conventional cosmological models (such as the horizon and flatness problems) could be explained by invoking inflationary models of the early universe with an exponential expansion phase at very early epochs. These models have the added advantage that they are able to make a definite prediction about the present matter density in the universe, i.e. they require that the density be exactly equal to the closure density which in turn can be easily estimated from the Hubble constant now known to within a factor of two. Now if one goes back to an earlier idea that explored the possibility of unusual clustering of quasar redshifts around $z=2$ or 3 , we get an example of another cosmological model with a definite prediction for the present overall matter density. This is a modified version of the Eddington-Lemaitre type of model which naturally accommodates such features as a clustering of quasars at certain epochs. From these models one can get a prediction for the present matter density which would be an involved function of the Hubble constant and the redshifts at which such clustering occurs. It can be shown that if such clustering had occurred at any $z$, the present matter density predicted would be substantially smaller than the corresponding closure density. The conclusion is that any clustering of quasar redshifts is incompatiable with inflationary universe models, indirectly providing observational support for these new theories.

The overall matter density of the universe is usually specified by the dimensionless parameter expressed as a ratio $\Omega=\rho / \rho_{c}$. Here $p$ is the actual density and $\rho_{c}$ is the so called critical density required to just close the universe. $\rho_{c}$ can be related to the measurable quantity called the Hubble constant $H_{O}$ as : $\rho_{c}=3 H_{O}^{2} / 8 \pi G$ where $H_{O}=(R / R)_{0}$ is the present value of the expansion rate of the universe, $R$ being the scale factor and $\dot{R}$ its time derivative, (o denoting present values) $G$ is the gravitational constant.

Now big bang nucleosynthesis of the elements deuterium (D), Helium $-4(\mathrm{He}-4), \mathrm{He}-3$, etc. as well as the dynamics of galaxies and clusters of galaxies are all consistent with $\Omega \simeq 0.1$ to 0.2 . However the conventional big bang model despite its success in naturally accounting for both the microwave background and the abundance of the light elements 
faces several serious problems when extrapolated to early epochs such as the horizon, flatness and monopole problems. Inflation models involving an early exponential expansion phase appear to resolve most of these problems. But these models require as a prediction that $\Omega=1$. One other model which makes a definite prediction for the present density $\rho_{0}$ of the universe is a variation of the Eddington-Lemaitre model which can also account for any clustering of quasar red shifts at any $z$ if indeed such clustering does occur. Such models involve a 'cosmological constant or $\Lambda$-term' which effectively increases the age of the universe thus :

$$
t_{H}=H_{0}^{-1} 0 \int^{\infty} \frac{d z}{(1+z)\left[(1+z)^{2}(1+\Omega z)-\left(\Lambda c^{2} / 8 \pi G \rho_{c}\right) z(2+z)\right]^{1 / 2}}
$$

If clustering of quasars had occurred at some $z$ (say $z=2.2$ ) when the scale factor of expansion was $R_{z}$ : then we obtain the following equations for the present scale factor, $\mathrm{R}_{0}, \mathrm{H}_{0}$ and density $\rho_{0}$ (all present values)

$$
\begin{aligned}
& R_{0}=R_{z}(1+z) ; R_{z} \sim \Lambda^{-1 / 2} \\
& H_{0}=(\dot{R} / R)_{0}=\left(\frac{1}{3} \Lambda c^{2}-\frac{c^{2}}{R_{0}^{2}}+\frac{2 c^{2}}{3 \Lambda^{\dot{1} / 2} R_{0}^{3 G}}\right)^{1 / 2} \\
& \rho_{0}=\left(4 \pi G R_{0}^{3} \Lambda^{1 / 2} / c^{2}\right)^{-1}
\end{aligned}
$$

For a given $z$ (where redshifts are thought to cluster), we can solve these equations to obtain both $\mathrm{H}_{0}$ and $\rho_{O}$. Thus for $z=2$, we have $\mathrm{H}_{\mathrm{O}}=(20 / 81 \Lambda)^{1} / \mathrm{C}$, and from the observed $\mathrm{H}_{\mathrm{O}}=50 \mathrm{~km} / \mathrm{s} / \mathrm{Mpc}$ we have the (predicted) $\rho_{0} \leq 5 \times 10^{-31} \mathrm{~g} / \mathrm{cc}<<\rho_{c}$. For $z=3, \mathrm{H}_{\mathrm{O}}=(27 / 96 \Lambda)^{1 / 2} \mathrm{C}$ again $\rho_{0} \ll \rho_{0}$. For all $z$ between 2 and 3 where observations have now and then reported clustering we have $\Omega<0.1$ as a prediction. As inflation requires $\Omega=1$, this is not in agreement with the presence of vast amounts of dark matter in the universe. 